\title{
An Unusual Cutaneous Presentation of Mycobacterium Tuberculosis Infection in a Renal Transplant Recipient
}

\author{
Authors \\ Dr N.Ashok Viswanath ${ }^{1}$, Dr Vasanthi Rompicherla ${ }^{2}$, Dr Priyadarshini Shanmugam ${ }^{3 *}$ \\ Department of Microbiology, Chettinad Hospital \& Research Institute, Chettinad Academy of Research \& Education, \\ Kelambakkam, Chennai, Tamilnadu, India \\ *Corresponding Author \\ Dr Priyadarshini Shanmugam \\ Professor \& Head, Department of Microbiology, Chettinad Hospital \& Research Institute
}

\begin{abstract}
A 30-year-old male, who had undergone renal transplantation from a deceased donor on august 2017 with a stable graft function, presented with the complaints of fever and an ulcer on the right foot. On examination, the patient was malnourished and febrile, on inspection the ulcer was irregular approximately $3 \times 2.5 \times 3 \mathrm{~cm}$ in size, situated on the lateral aspect of dorsum of the right foot with yellowish white discharge, creamy in consistency. Pus for culture and sensitivity revealed the growth of Enterobacter spp which was sensitive to almost all the antibiotics used for Gram negative bacilli. Ziehl-Neelsen method of staining by using $25 \%$ sulfuric acid as a decoloriser showed the presence of acid-fast bacilli in all smears. The pus sample was sent for Cartridge Based Nucleic Acid Amplification (Gene Xpert) test which was positive for Mycobacterium tuberculosis with negative rifampicin resistance.
\end{abstract}

Keywords: Rare presentation, skin tuberculosis, Mycobacterium, Gene Xpert.

\section{Introduction}

Cutaneous tuberculosis is a rare disease, but prevalent among high risk population in endemic regions. It usually affects individuals with secondary immunodeficiency states like HIV infection, patients on immunosuppressive therapy and protein energy malnutrition. Usually tuberculosis presents as a pulmonary, intestinal, spinal, involving bones in the form of osteomyelitis with or without multiple draining sinuses ${ }^{(1)}$.

The clinician may misdiagnose cutaneous tuberculosis with either non-healing diabetic ulcers, venous and arterial ulcers or pressure ulcers.

However, they contribute to morbidity and mortality, when proper diagnosis is missed, increasing the of risk-factor while screening for acid fast organisms in the wound swabs.

\section{Case Report}

A 30-year-old male, native of Chennai, Tamilnadu, had undergone a renal transplantation in August 2017 with a stable graft function, he was put on immunomodulators. After few days he presented with the complaints of continuous high-grade fever, along with a non healing ulcer on the lateral aspect of dorsum of the right foot. On examination, the patient was malnourished, febrile, with a tender swelling around the ulcer. Patient was hemodynamically stable; no abnormalities were noted on systemic examination.

A diagnosis of cellulitis with ulcer was made and he was started on intravenous cefotaxime $1 \mathrm{gm}$ twice daily for seven days, the symptoms subsided. After one month he again developed fever with an intensely tender ulcer. The ulcer was approximately 
$3 \times 2.5 \times 3 \mathrm{~cm}$ in size on the lateral aspect of dorsum of right foot with yellowish white discharge, creamy in consistency, with granulation tissue and slough, the surrounding areas showed the evidence of inflammation (Fig.1). Routine laboratory workup revealed pancytopenia, increased erythrocyte sedimentation rate. Neither history of past pulmonary disease nor history of contact with tuberculosis was reported.

Under aseptic precaution pus was collected from the ulcer using a sterile cotton tipped swab and subjected to microbiological analysis. Routine Gram staining revealed numerous pus cells with numerous Gram-negative bacilli. Aerobic bacterial culture yielded heavy growth of Enterobacter species sensitive to all the antibiotics for Gram negative panel. Ziehl Neelsen staining (Fig.2) of the same sample with sulphuric acid at the concentrations of $1 \%, 5 \%, 25 \%$ was used as a decolouriser, revealed the presence of acid-fast bacilli confirming the diagnosis of cutaneous tuberculosis.

The sample was subjected to fluorescent staining which revealed fluorescent green slender bacilli against dark background (Fig.3). Nucleic acid amplification study analysis by Gene Xpert (Fig.4) at Regional Tuberculosis Centre was positive for Mycobacterium tuberculosis with no resistance to rifampicin.
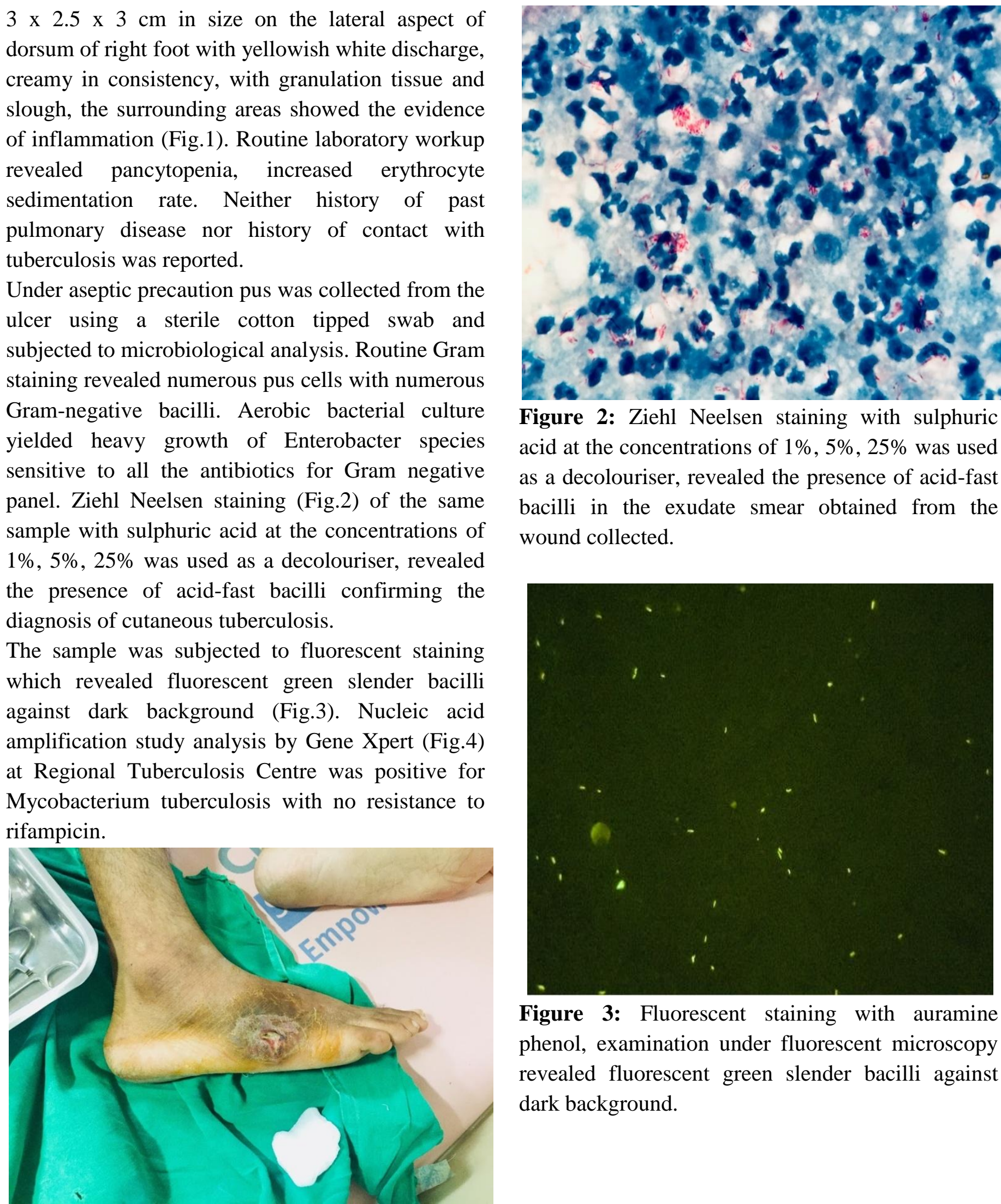

Figure 2: Ziehl Neelsen staining with sulphuric acid at the concentrations of $1 \%, 5 \%, 25 \%$ was used as a decolouriser, revealed the presence of acid-fast bacilli in the exudate smear obtained from the wound collected.

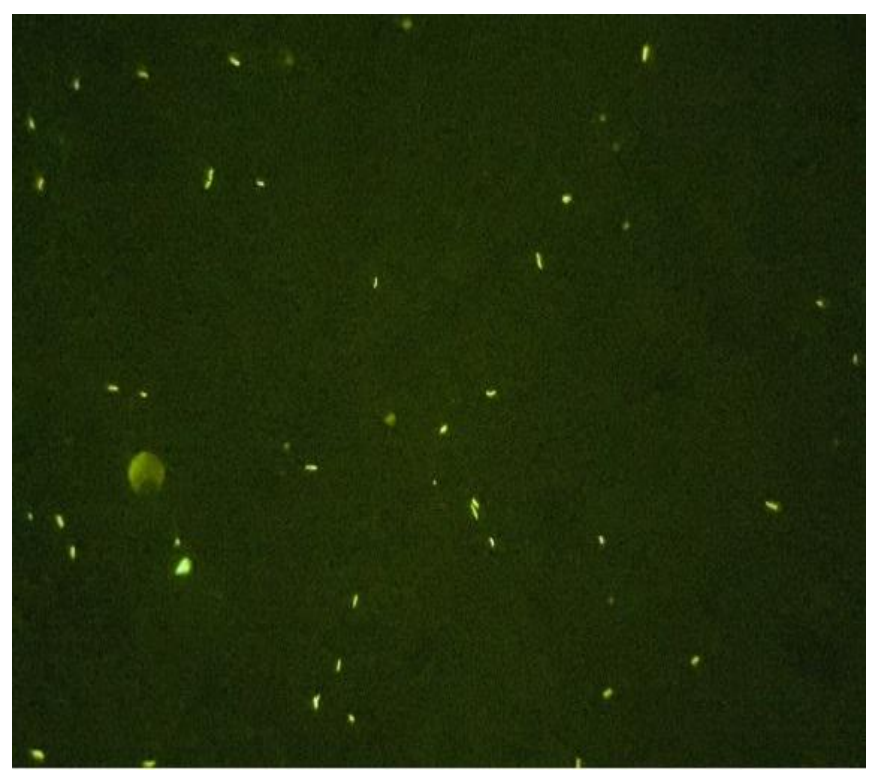

Figure 3: Fluorescent staining with auramine phenol, examination under fluorescent microscopy revealed fluorescent green slender bacilli against dark background.

Figure 1: An Irregular ulcer on the lateral aspect of dorsum of right foot with yellowish white discharge, creamy in consistency, with granulation tissue and slough, the surrounding areas showed the evidence of inflammation 


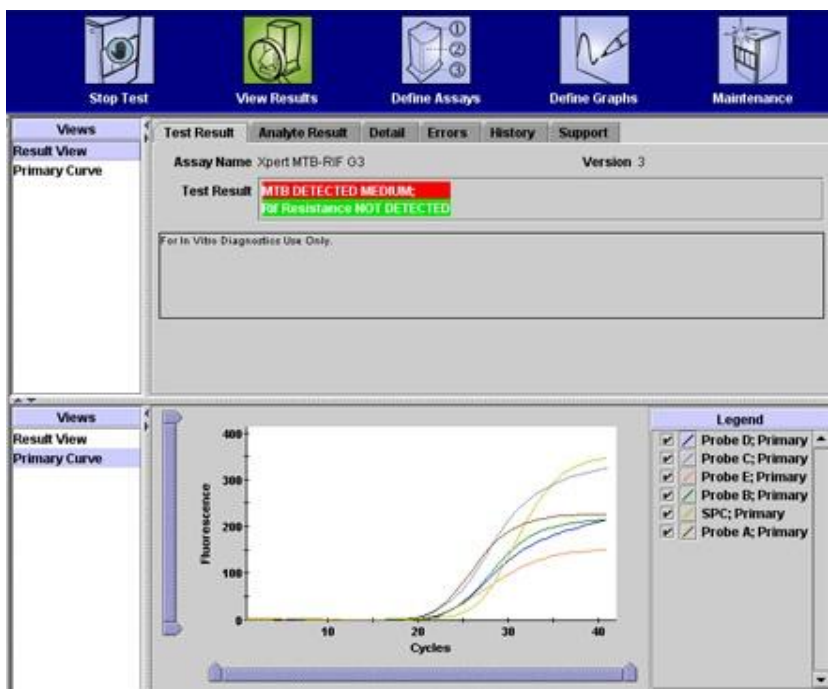

Figure 4: Nucleic acid amplification study analysis by GeneXpert - a catridge based nucleic acid amplification technique - Positive for Mycobacterium tuberculosis without Rifampicin resistance.

\section{Discussion}

In 2013, about 5.7 million newly diagnosed cases were notified to national TB programs, and in 2016 about 10.4 million people fell ill with TB, and 1.7 million died from the disease (including 0.4 million among people with co-infection of HIV). Over $95 \%$ of deaths due to tuberculosis occurred in low- and middle-income countries. Seven countries account for about $64 \%$ of the total cases, among them India is leading, followed by Indonesia, China, Philippines, Pakistan, Nigeria, and South Africa as per $\mathrm{WHO}^{(2)}$. In general, $\mathrm{TB}$ is diagnosed by identification of Mycobacterium tuberculosis by Ziehl Neelsen staining in about $30 \%$ to $40 \%$ of cases. A high index of suspicion is the key for successful diagnosis and treatment, especially in immunocompromised individuals living in countries with a high disease burden. A combination of staining and molecular methods is useful in diagnosis and for further evaluation of patients with non-healing ulcers. Mantoux test is inconclusive in tropical countries and is not helpful, especially in immunosuppressed individuals $^{(3)}$. On the other hand, the tuberculous ulcers are generally indistinguishable from other non-healing ulcers. The gold standard for the diagnosis of cutaneous tuberculosis is detection of Mycobacterium species by Ziehl Neelsen staining or by culture. Differential acid-fast staining on the exudate smears detects the presence of acid-fast organisms, leading to definitive diagnosis. Nucleic acid amplification test (GeneXpert) is highly sensitive and confirmatory method for the diagnosis ${ }^{(4)}$. Although not routinely used, it is recommended in cases with negative culture results or for differential diagnosis between Mycobacterium tuberculosis and other mycobacteria.

\section{Conclusion}

Active tuberculosis presenting as a non-healing ulcer is not uncommon. Patients with non-healing ulcers presenting for the first time especially those who are immunocompromised due to several factors should be investigated thoroughly. There are no specific clinical pointers towards likelihood of TBinduced non-healing ulcers and only a high index of suspicion with relevant tests and its treatment, would aid in early diagnosis and prevent complications in these patients.

\section{References}

1. Sriram K, Moffatt D, Stapledon R. Tuberculosis infection of the breast mistaken for granulomatous mastitis: a case report. Cases Journal 2008;1:273.

2. Fox W, Ellard GA, Mitchison DA. Studies on the treatment of tuberculosis undertaken by the British Medical Research Council tuberculosis units, 1946-1986, with relevant subsequent publications. Int J Tuberc Lung Dis. 1999;3(10 Suppl 2):S231-79.

3. Menzies D. Tuberculin skin testing. In: Reichman LB, Hershfield ES, editors. Tuberculosis: A comprehensive international approach. New York: Marcel Dekker; 2000. pp. 279-322.

4. TB INDIA 2013, Revised National TB control Programme Annual status report.

5. WHO global tuberculosis report 2014.

6. Centers of Disease Control and Prevention. Treatment of tuberculosis. MMWR. 2003;52 (RR-11):1-77. 